\title{
Affinity Propagation for Class Exemplar Mining
}

\author{
Shengping Xia ${ }^{1, \star}$, Rui Song ${ }^{1}$, and Edwin R. Hancock ${ }^{2, \star \star}$ \\ 1 ATR Lab, School of Electronic Science and Engineering, National University of Defense \\ Technology, Changsha, Hunan, P.R. China 410073 \\ 2 Department of Computer Science, University of York, York YO1 5DD, UK
}

\begin{abstract}
This paper focusses on the problem of locating object class exemplars from a large corpus of images using affinity propagation. We use attributed relational graphs to represent groups of local invariant features together with their spatial arrangement. Rather than mining exemplars from the entire graph corpus, we prefer to cluster object specific exemplars. Firstly, we obtain an object specific cluster of graphs using a similarity propagation based graph clustering (SPGC) method. Here a SOM neural net based tree clustering method is used to incrementally cluster a large corpus of local invariant descriptors. The popular affinity propagation based clustering algorithm is then individually applied to each object specific cluster. Using this clustering method, we obtain object specific exemplars together with a high precision for the data associated with each exemplar. The strategy adopted is one of divide and conquer, and this greatly increases the efficiency of mining exemplars. Using the exemplars, we perform recognition using a majority voting strategy that is weighted by nearest neighbor similarity. Experiments are performed on over 80K images spanning 500 objects, and demonstrate the performance in terms of efficiency, scalability and recognition.
\end{abstract}

\section{Introduction}

One of the most effective means of knowledge discovery from a large corpus of data is to search for class exemplars. Detecting exemplars goes beyond simple clustering, as the exemplars themselves store compressed information. Frey and Dueck [1] propose an affinity propagation method for locating an optimal set of exemplars. Each data item in the corpus is then associated with the exemplar that best represents it. Affinity propagation uses an index of similarity $s(i, k)$ to indicate how well the data item with index $k$ is suited to be the exemplar for data item $i$. Affinities are updated in an iterative manner reminiscent of both belief propagation and relaxation labeling. The method can be applied to high dimensional vectors, graphs or any data-structure provided a suitable similarity measure can be defined.

Many knowledge discovery tasks require the identification of exemplars from among a set of sparsely related data, i.e., where most similarities are either unknown or large and negative. To deal with affinity propagation in this case, a sparse similarity matrix

\footnotetext{
* Supported by Chinese NSF Foundation project (contract 60972114).

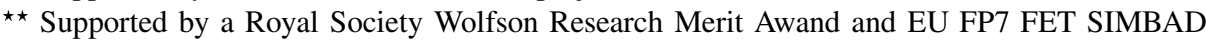
project (contract 213250).
}

E.R. Hancock et al. (Eds.): SSPR \& SPR 2010, LNCS 6218, pp. 191-201, 2010.
(c) Springer-Verlag Berlin Heidelberg 2010 
(SSM) with similarity set to $-\infty$ is used. When affinity propagation is applied to this sparse similarity matrix, because messages need not be exchanged between $i$ and $k$ if $\mathrm{s}(i, k)=-\infty$, each iteration requires exchanging messages only between a very small subset of the data pairs. Another interesting capability of affinity propagation is that is can be applied to asymmetric or non-metric similarities (i.e. those for which $s(i, k) \neq$ $s(k, i))$ and those for which the similarities do not satisfy the triangle inequality (i.e., $s(i, k)<s(i, j)+s(j, k))$.

The affinity propagation method has been applied to a moderately large image corpus [1]. However, it is still difficult to apply the algorithm to a very large sparse similarity matrix, for instance where the number of data items exceed $10^{7}$. Furthermore, it is difficult to efficiently obtain a sparse similarity matrix for large datasets, and this can cause difficulties in locating exemplars. In this paper we address the question of how to obtain exemplars of a specific object rather than all exemplars of a large image corpus, and how to propagate affinity in this context.

Recently, Xia and Hancock have shown how graph clustering can be effected using similarity propagation and used to discover the set of object classes present in a database of images[7][6][4]. In this work, each image of an object is represented by a graph constructed from a selected group of robust SIFT features. For each pair of graphs, a similarity measure is computed using the cardinality of the maximum common subgraph and the consistency of geometric spatial alignment of the image features. A recursive self organizing map is used to locate a clustering tree (termed RSOM) for the SIFT descriptors, which is then incrementally trained using the method outlined in [2]. For each graph, the $\mathrm{K}$ nearest neighbor graphs under the pairwise graph similarity measure can be efficiently located using the RSOM clustering tree.

In this paper, we propose an integrated framework for obtaining object specific image exemplars based on the more principled use of affinity propagation. The paper is organized as follows. In Section 2, we introduce some preliminaries for our work. In Section 3, we present the outline of our method used for exemplar mining. We present experimental results in Section 4 and conclude the paper in Section 5.

\section{Ingredients of Our Method}

\subsection{Image Representation}

For each image in the dataset local invariant features are detected. A variety of feature detectors have been developed [8][14][9][15], and these include SIFT [9] and SURF ( Speeded Up Robust Features ) [8]. We use the method proposed in [5] to extract a selected number $\mathcal{T}$, e.g. $\mathcal{T}=40$, of salient SIFT features. Each group of selected local features together with their spatial arrangement is regarded as a semantic visual entity. This kind of structured data can be represented by using attributed graphs $G$ [11] (hereafter simply graphs). We can obtain a set of graphs $\mathbb{G}=\left\{G_{l}, l=1,2, \ldots, N\right\}$ from a set of images.

\subsection{Pairwise Graph Matching}

As shown in [10][16][20], the recognition or retrieval results can be significantly improved using the geometry of spatial feature arrangement to verify consistency. In our 
approach, on the other hand, each image is represented by a graph. As a result the spatial verification problem becomes one of pairwise graph matching (PGM). We perform PGM with the aim of finding a maximum common subgraph (MCS) between two graphs $G_{l}$ and $G_{q}$, and the result is denoted as $\operatorname{MCS}\left(G_{l}, G_{q}\right)$. There are a plethora of available methods for finding matching features consistent with a given set of geometric constraints, and the problem has been proven to be NP-hard. RANSAC provides one popular set of methods, however their implementation is slow [18]. In [3], pairwise graph matching is achieved by combining SIFT feature matching and iterative Procrustes alignment [19]. The method can not only be used to align the feature points, but can also be used to discard those features that do not satisfy the spatial arrangement constraints. Given the $\operatorname{MCS}\left(G_{l}, G_{q}\right)$ obtained by PGM, a similarity measure between the graphs $G_{l}$ and $G_{q}$ is defined as follows:

$$
R\left(G_{l}, G_{q}\right)=\left\|\operatorname{MCS}\left(G_{l}, G_{q}\right)\right\| \times\left(\exp \left(-e\left(X_{l}, X_{q}\right)\right)\right)^{K} .
$$

Here a) $\left\|\operatorname{MCS}\left(G_{l}, G_{q}\right)\right\|$ is the cardinality of the MCS of $G_{l}$ and $G_{q}$, b) $\kappa$ is the number of roughly mismatched feature pairs by SIFT matching, which is used to amplify the influence of the geometric dissimilarity between $X_{l}$ and $X_{q}$, and c) $X_{l}$ and $X_{q}$ are respectively the position coordinates in graphs $G_{l}$ and $G_{q}$ corresponding to the vertices of $\operatorname{MCS}\left(G_{l}, G_{q}\right)$.

\subsection{Obtaining K-Nearest Neighbors Using RSOM Tree}

Consider the graph set $\mathbb{G}=\left\{G_{q}, q=1,2, \ldots, N\right\}$. For each graph $G_{l} \in \mathbb{G}$, and the remaining graphs in the set $\left(\forall G_{q} \in \mathbb{G}\right)$, we obtain the pairwise graph similarity measures $R\left(G_{l}, G_{q}\right)$ using Equation (1). Using the similarity measures we rank the graphs in descending order of similarity and the K top-ranked graphs are defined as the generalized K-nearest neighbor graphs (KNNG) of graph $G_{l}$, denoted as $\mathbb{K}\left\{G_{l}\right\}$.

With increasing size of the graph dataset, it becomes time consuming to obtain all $\mathbb{K}\left\{G_{l}\right\}$ if a sequential search strategy is adopted. However, in a large graph set, most of the values of the the similarity measures are very low. For a single graph $G_{l}$, if we can efficiently find a subset $\mathbb{G}^{\prime}$ with significant similarity values from the complete set $\mathbb{G}$ as a filtering stage. Then we only need to perform pairwise graph matching for this subset. To this end, we employ a tree based clustering method.

We use the incremental clustering tree-RSOM reported in [2] for incrementally learning a large corpus of SIFT descriptors. To obtain $\mathbb{K}\left\{G_{l}\right\}$ for each training graph using a trained RSOM tree we proceed as follows. Given a graph $G_{l}$, we find the winner of the leaf nodes for each descriptor of this graph and define the union of all graphs for the winners as follows:

$$
U G\left\{G_{l}\right\}=\left\{G_{q} \mid U_{q}^{j} \in G_{q}, U_{q}^{j} \in W L\left\{U_{l}^{t}\right\}, U_{l}^{t} \in G_{l}\right\}
$$

where $W L\left\{U_{l}^{t}\right\}$ is the winner of the leaf nodes for descriptor $U_{l}^{t}$. The frequency of graph $G_{q}$, denoted as $H_{q}$, represents the number of roughly matched descriptors between two graphs. Since we aim to obtain $\mathbb{K}\left\{G_{l}\right\}$, we need not process all graphs in the subsequent stages. We rank the graphs in $U G\left\{G_{l}\right\}$ according to decreasing frequency $H_{q}$ of graph 
$G_{q}$. From the ranked list, we select the first $K$ graphs, denoted by $\mathbb{K}^{\prime}\left\{G_{l}\right\}$ as follows:

$$
\mathbb{K}^{\prime}\left\{G_{l}\right\}=\left\{G_{q} \mid G_{q} \in U G\left\{G_{l}\right\}, H_{q}>H_{q+1}, q=1,2, \ldots, K .\right\} .
$$

For each graph $G_{q}$ in $\mathbb{K}^{\prime}\left\{G_{l}\right\}$, we will obtain the similarity measure according to Equation (1) and then $\mathbb{K}\left\{G_{l}\right\}$ can be obtained.

\subsection{Similarity Propagation Based Graph Clustering(SPGC)}

In the text retrieval literature, a standard method for improving performance is query expansion. The query expansion strategy used in [4] is based on the RSOM tree and the set $\mathbb{K}\left\{G_{l}\right\}$ for each graph, obtained in the training stage. Stated simply, the method is as follows. A group of graphs are referred to as siblings of a given graph $G_{l}$ provided they satisfy the following condition:

$$
S\left\{G_{l}\right\}=\left\{G_{q} \in \mathbb{K}\left\{G_{l}\right\} \mid R\left(G_{l}, G_{q}\right) \geq \tau\right\} \triangleq S_{\tau}\left\{G_{l}\right\} .
$$

where $\tau$ is a similarity threshold. We use the definition to recursively obtain the family tree for the graph $G_{l}$, and this is formally defined as follows.

Family Tree of a Graph (FTOG): For any given similarity threshold $\tau$, an FTOG of $G_{l}$ with $k$ generations and denoted as $M\left\{G_{l}, k\right\}$, is defined as follows:

$$
M\left\{G_{l}, k\right\}=M\left\{G_{l}, k-1\right\} \bigcup_{G_{q} \in L\left\{G_{l}, k-1\right\}} S_{\tau}\left\{G_{q}\right\} .
$$

where, if $k=1, L\left\{G_{l}, 1\right\}=L\left\{G_{l}, 0\right\} \cup S\left\{G_{l}\right\}$ and $M\left\{G_{l}, 0\right\}=\left\{G_{l}\right\}$; and the process stops when $M\left\{G_{l}, k\right\}=M\left\{G_{l}, k+1\right\}$. An FTOG, whose graphs satisfy the restriction defined in Equation (4), can be regarded as a cluster of graphs. However, it must be stressed that this is not a clustering method based on a central prototype.

\subsection{Affinity Propagation Clustering}

Affinity propagation is a clustering method, which commences by considering all the data items as potential exemplars, and then recursively transmits real-valued messages along edges of a network whose nodes are data items. At any item and at any time, the magnitude of each message reflects the current affinity (or support) provided by one node for another as its potential exemplar [1]. After a number of iterations, a good set of exemplars and corresponding clusters emerges. The input of affinity propagation is a collection of real-valued similarities between data items, where the similarity $s(i, k)$ indicates how well data point $k$ is suited as the exemplar for data-point $i$. In affinity propagation, the number of clusters is not required to be specified. The method can be biassed by adjusting values of $s(i, i)$ referred to as the "preference". A data point with a large value of $s(i, i)$ is more likely to be chosen as an exemplar. Two kinds of messages are exchanged between data items, namely "responsibility" and "availability". The "responsibility" $r(i, k)$, transmitted from item $i$ to item $k$, reflects how well-suited data item $k$ is to serve as the exemplar for data item $i$. The "availability" $a(i, k)$, transmitted from candidate exemplar item $k$ to item $i$, reflects the accumulated evidence for choosing item 
$k$ as the exemplar of item $i$, given the support from the remaining items in the network. Updating takes place according to the following rules:

$$
\left\{\begin{array}{l}
r(i, k):=s\left(x_{i}, x_{k}\right)-\max _{k^{\prime} \neq k}\left\{a\left(i, k^{\prime}\right)+s\left(x_{i}, x_{k^{\prime}}\right)\right\} \\
a(i, k):=\min \left\{0, r(k, k)+\sum_{i^{\prime} \sim \in\{i, k\}} \max \left\{0, r\left(i^{\prime}, k\right)\right\}\right\}
\end{array}\right.
$$

The self-availability is updated in a slightly different way as

$$
a(k, k)=\sum_{i^{\prime} \neq k} \max \left\{0, r\left(i^{\prime}, k\right)\right\} .
$$

Upon convergence, the exemplar for the data item indexed $i$ is chosen as $e\left(x_{i}\right)=x_{k}$ where $k$ satisfies the criterion:

$$
k=\arg \max _{k}\{a(i, k)+r(i, k)\}
$$

The algorithm is halted after a fixed number of iterations or after the exemplars do not change for a given number of iterations. Affinity propagation is not a universally efficient data clustering method. Firstly, if the desirable number of clusters $\mathrm{K}$ is small, then the combinatorial problem can be tackled by brute force (considering all $N^{K}$ possible solutions). Secondly, and most importantly, affinity propagation suffers from quadratic computational complexity in the number of data items $N$. This hinders its direct use in large-scale applications. To reduce the computational complexity of affinity propagation, in this paper we proposed an algorithm based on similarity propagation based clustering. We split the entire dataset into subsets of graphs, and then perform exemplar extraction on each subset.

\section{Mining Exemplars Using Three Stage Clustering}

Suppose that we have obtained a large graph set $\mathbb{G}=\left\{G_{l}, l=1,2, \ldots, N\right\}$, extracted from an image corpus. In this section, we demonstrate how to efficiently obtain object specific exemplars from such datasets. Our method involves four main steps:

- Step 1 Train the RSOM tree clustering of SIFT descriptors;

- Step 2 Obtain the KNNG for each graph in $\mathbb{G}$;

- Step 3 Obtain the FTOG in a weakly supervised manner using SPGC;

- Step 4 Detect exemplars for each FTOG individually using affinity propagation.

Steps 1-3 follow directly the work reported in [4]. In Step 4, we firstly apply affinity propagation to each FTOG and rank all graphs according to $\arg \max _{k}\{a(i, k)+r(i, k)\}$. We select $J$ exemplars according to Equation 8 and check whether these $J$ exemplars form an FTOG. If not, we select the $J^{\prime}>J$ top ranked graphs such that these selected graphs form an FTOG. In this way, we simplify each FTOG $M_{l}$ to $M_{l}^{\prime}$ which is constructed from a group of exemplar graphs $G_{j}^{l}, j=1,2, \ldots,\left\|M_{l}^{\prime}\right\|$ and their similarity relationships. 
For each feature point as a node $k$ of an exemplar graph $G_{j}^{l}$ in the FTOG $M_{l}^{\prime}$, we count the frequency $n_{j k}^{l}$ of being matched by its nearest neighbors. The simplified FTOG $M_{l}^{\prime}$ depends on $M_{l}$ and $R_{0}$ and is a more compact representation.

In this way a compact representation of each FTOG is obtained. The set of compact FTOGs forms the learned model of the object of interest. Once the object model is trained, for a test graph $G_{l}$, we can obtain $K\left\{G_{l}\right\}$ and use a weighted voting method based on k-nearest neighbor graphs for recognition, where the similarity measure $R\left(G_{l}, G_{q}\right)$ is used as the weight. We then use the well known F-measure in order to evaluate the recognition performance. A high value of the F-measure $f$ means that both high recall and high precision are achieved. The ideal result is $f=1$.

\section{Experimental Results}

\subsection{Datasets}

We have collected 53,536 images as a training set. This dataset spans more than 500 objects, including some human faces and natural scenes. The image corpus is composed as follows: there are a) 3600 images of 50 objects from COIL 100, labeled A1 A50; b) 161 images of 8 objects used in [18], labeled B1 to B8; c) 20000 images of 10 objects collected in our own lab., labeled as $\mathrm{C} 1$ to $\mathrm{C} 10$. For each of the objects in $\mathrm{C} 1$ to $\mathrm{C} 9$, we have collected 1500 images which traverse large variations of imaging conditions, and similarly 6500 images for C10; d) 29875 unlabeled images from many other standard datasets, e.g. Caltech101 [13], PASCAL VOC'07 [12] and Google image, spanning over 450 objects and used as negative samples. For simplicity, the 4 data sets are denoted as A, B, C and D. The objects in Figure 12 and 3 are numbered from left to right and then from top to bottom as shown in the corresponding figures. We take 68 images as object examples for recognition, and these are identified as Object 1 to Object 68 in Figure 1 , 2 and 3 .

For each of these images, we extract ranked SIFT features, using the method presented in [5], of which at most 40 highly ranked features are selected to construct a graph.

\subsection{Clustering Results}

From the training data we have obtained an RSOM clustering tree with 25334 leaf nodes using the method described in [2]. The method was implemented using Matlab 7.2 and run on a $2.14 \mathrm{GHz}$ computer with $2 \mathrm{G}$ RAM. In the incrementally training process, we have obtained $K\left\{G_{l}\right\}$ for each of the graphs.

Following RSOM tree clustering, we individually obtain FTOG's for the above 68 labeled object classes using the similarity propagation based graph clustering method presented in Section 2 The object clustering results for the 68 object problem are shown in Figure 5. For most of the objects sampled under controlled imaging conditions, ideal performance has been achieved. For 35 objects in COIL 100, 35 models are individually clustered with total unit recall and precision in one FTOG. For 13 objects, 6 FTOGs are obtained. Each group of objects in Figure 6(A)(B)(C)(D) are actually identical in shape but color. Since it only uses gray scale information in SIFT, our method fails in this case. 


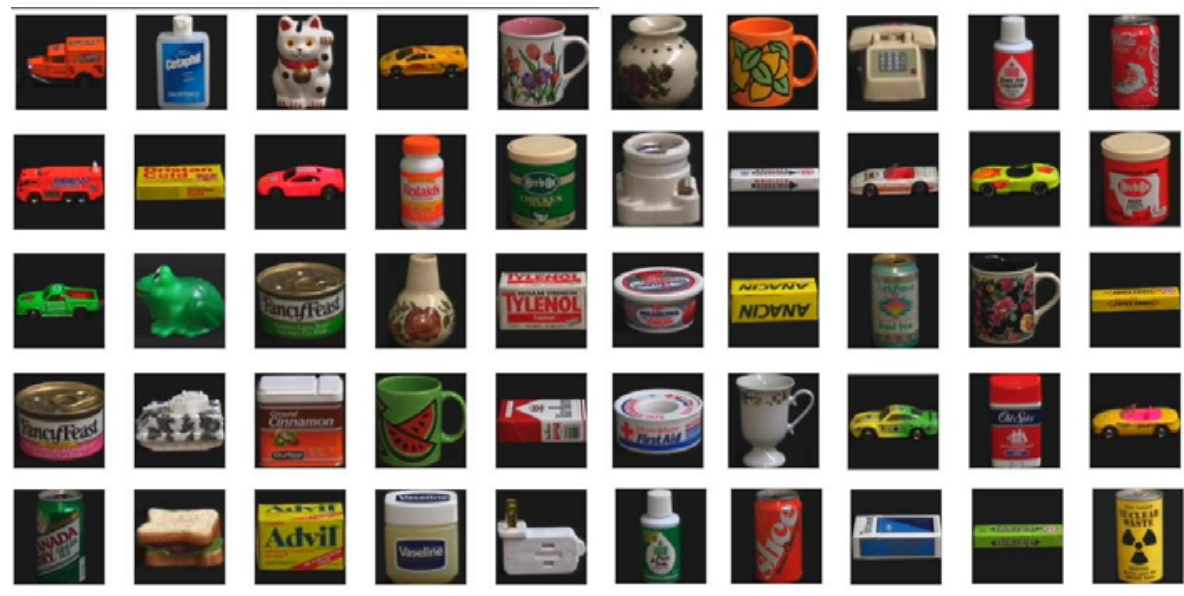

Fig. 1. 50 objects in Coil 100



Fig. 2. 8 objects in 18

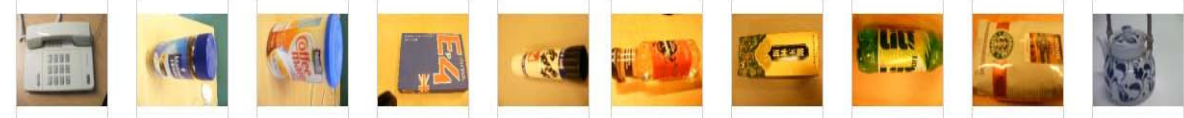

Fig. 3. 10 objects collected by the authors

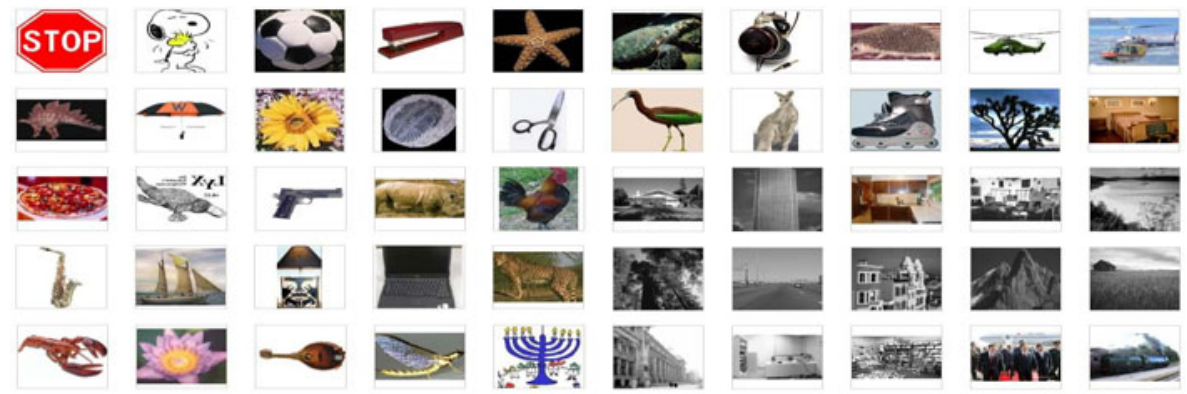

Fig. 4. Unlabeled sample images 


\begin{tabular}{|c|c|c|c|c|c|c|c|c|c|c|c|c|c|c|c|c|c|c|c|c|c|}
\hline ID & $\begin{array}{c}1 \sim 50 \\
\text { Par }\end{array}$ & 3 & 39 & 51 & 52 & 53 & 54 & 55 & 56 & 57 & 58 & 59 & 60 & 61 & 62 & 63 & 64 & 65 & 66 & 67 & 68 \\
\hline$N_{f}$ & 72 & 72 & 72 & 29 & 20 & 16 & 16 & 16 & 16 & 28 & 20 & 1500 & 1500 & 1500 & 1500 & 1500 & 1500 & 1500 & 1500 & 1500 & 6500 \\
\hline$N_{d}$ & 72 & 64 & 66 & 22 & 16 & 15 & 15 & 16 & 16 & 27 & 20 & 1491 & 1483 & 1500 & 1500 & 1500 & 1475 & 1487 & 1500 & 1467 & 6488 \\
\hline$p$ & 1.0 & 1.0 & 1.0 & 1.0 & 1.0 & 1.0 & 1.0 & 1.0 & 1.0 & 1.0 & 1.0 & 1.0 & 1.0 & 1.0 & 1.0 & 1.0 & 1.0 & 1.0 & 1.0 & 1.0 & 1.0 \\
\hline$r$ & 1.0 & .875 & 917 & .759 & .80 & .938 & 938 & 1.0 & 1.0 & 964 & 1.0 & 994 & 989 & 1.0 & 1.0 & 1.0 & 983 & 991 & 1.0 & 978 & 998 \\
\hline$N_{\varepsilon}$ & 1 & 2 & 2 & 3 & 1 & 3 & 3 & 3 & 3 & 4 & 2 & 6 & 8 & 4 & 5 & 3 & 9 & 3 & 3 & 4 & 1 \\
\hline
\end{tabular}

Fig. 5. Results of object clustering using similarity based graph clustering. In the above table, ID is the Object ID; $N_{i}$ is the number of the initial images of an object; $N_{d}$ is the number of images clustered by using similarity based graph clustering; $N_{d}^{+}$is the number of correctly clustered images; $p$ is the precision defined as $N_{d}^{+} / N_{d} ; r$ is recall defined as $N_{d}^{+} / N_{i} . N_{c}$ is the number of clusters for each object.

(A)

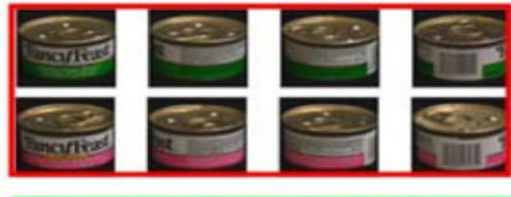

(B)

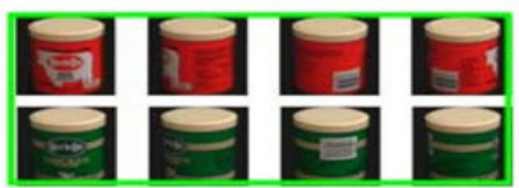

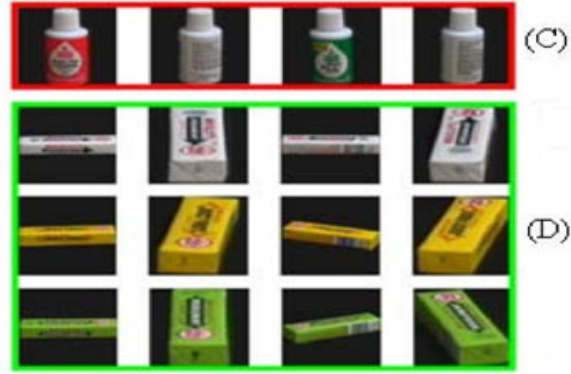

(D)

Fig. 6. 6 groups of objects are overlapping-clustered into 6 clusters

We hence regard these objects in the four groups as being correctly clustered according to shape.

Unfortunately, in most practical situations, the images of an object are likely to be obtained with large variations of imaging conditions and are more likely to be clustered into several FTOGs. As a result, each object gives rise to multiple clusters. For objects 51 to 58 there are more than 30 images with large variations in viewing conditions, and the images are not representative enough to perform ideal recognition. However, for objects 59 to 68 , the images clustered together are sufficient to form an effective object model which can be used for recognition. For object 68 , since there are thousands of images, the different views form a single cluster.

For each FTOG, the exemplar graphs are obtained individually using affinity propagation. The percentage of the exemplar graphs for each object are shown in Figure 7.

\subsection{Recognition Test Results}

We also collect images of Objects 1 to 68 for recognition experiments. For each of objects A1 to A50 (i.e. those contained in the COIL database), we synthesis 6 images by adding a mixture of salt and pepper noise, speckle noise and Gaussian noise to their original image. The variances of the noise processes are randomly set to $0.03,0.04$ or 0.05. In total, 21600 images of the 50 objects are obtained. For objects B1 to B8, we 

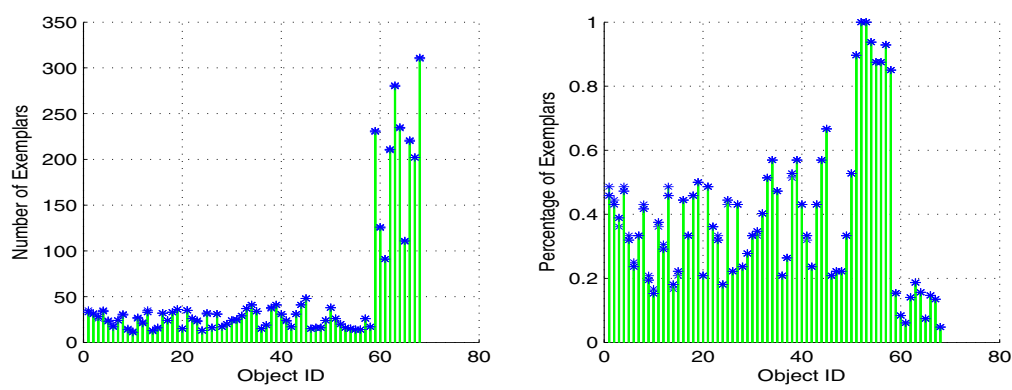

Fig. 7. The numbers and percentages of exemplars obtained by using SPCG and affinity propagation for the objects of interest

Table 1. F-measure $f$ for given test set of Object 1 68

\begin{tabular}{|c|c|c|c|c|c|c|c|c|c|c|c|c|c|c|}
\hline D $f$ & \begin{tabular}{|l|l} 
ID & $f$ \\
\end{tabular} & \begin{tabular}{|l|l|} 
IID & $f$ \\
\end{tabular} & \begin{tabular}{|l|l|} 
ID & $f$ \\
\end{tabular} & \begin{tabular}{|l|l|} 
ID & $f$ \\
\end{tabular} & ID & $\bar{f}$ & IID & $f$ & HO & $\bar{f}$ & IID & $\bar{f}$ & ID & $f$ \\
\hline \begin{tabular}{l|l}
1.997 \\
\end{tabular} & \begin{tabular}{||l|l|} 
& 2 \\
\end{tabular} & \begin{tabular}{|l|l|}
3 & 1.0 \\
\end{tabular} & \begin{tabular}{|l|l|}
4 & .984 \\
\end{tabular} & \begin{tabular}{||l|l|}
5 & 1.0 \\
\end{tabular} & 6 & 1.0 & 7 & 1.0 & & 990 & 9 & 1. & $1 / 10$ & \begin{tabular}{|l|}
1.0 \\
\end{tabular} \\
\hline 1.983 & \begin{tabular}{|l|l|}
12 & 1.0
\end{tabular} & \begin{tabular}{|l|l|}
13.988 \\
\end{tabular} & \begin{tabular}{|l|l|}
14 & 1.0 \\
\end{tabular} & \begin{tabular}{||l|l|}
15 & 1.0 \\
\end{tabular} & $\mid 16$ & 1.0 & 17 & 1.0 & & 982 & 12 & 981 & 150 & 1.0 \\
\hline 1.986 & \begin{tabular}{l|l}
22 & $\mathbf{1 . 0}$
\end{tabular} & \begin{tabular}{|l|l|}
23 & 1. \\
\end{tabular} & \begin{tabular}{|l|l|}
24 & 1.0 \\
\end{tabular} & \begin{tabular}{|l|l|}
25 & 1.0 \\
\end{tabular} & 26 & 1.0 & 27 & 993 & 28 & 1.0 & 29 & 1. & 30 & $\mid 1.0$ \\
\hline \begin{tabular}{|l|l|}
31 & $\mathbf{1 . 0}$ \\
\end{tabular} & \begin{tabular}{|l|l|}
32 & $\mathbf{1 . 0}$ \\
\end{tabular} & \begin{tabular}{|l|l|}
33.994 \\
\end{tabular} & \begin{tabular}{|l|l|}
34 & $\mathbf{1 . 0}$ \\
\end{tabular} & \begin{tabular}{|l|l|}
35 & $\mathbf{1 . 0}$ \\
\end{tabular} & 36 & 1.0 & 37 & \begin{tabular}{|l|}
1.0 \\
\end{tabular} & 38 & 995 & 39 & 980 & 40 & \begin{tabular}{|l|}
1.0 \\
\end{tabular} \\
\hline \begin{tabular}{|l|l|}
41 & $\mathbf{1 . 0}$ \\
\end{tabular} & |420.988 & \begin{tabular}{|l|l|}
43 & 1.0 \\
\end{tabular} & 44.995 & \begin{tabular}{|l|l|}
45.941 \\
\end{tabular} & 46 & 1.0 & 47 & 1.0 & 48 & 1.0 & 49 & 1.0 & 5( & 989 \\
\hline 51.625 & \begin{tabular}{||l|l|}
52 & $\mathbf{1 . 0}$
\end{tabular} & \begin{tabular}{|l|l|}
53 & $\mathbf{1 . 0}$ \\
\end{tabular} & \begin{tabular}{|l|l|}
54 & $\mathbf{1 . 0}$ \\
\end{tabular} & \begin{tabular}{||l|l|}
55.714 \\
\end{tabular} & 56 & 1.0 & 57 & 954 & 58 & 1.0 & 59 & 99 & 60 & 998 \\
\hline \begin{tabular}{|l|l|}
51.625 \\
\end{tabular} & \begin{tabular}{|l|l|}
52 & $\mathbf{1 . 0}$ \\
\end{tabular} & \begin{tabular}{|l|l|}
53 & $\mathbf{1 . 0}$ \\
\end{tabular} & \begin{tabular}{|l|l|}
54 & 1.0 \\
\end{tabular} & 55.714 & 56 & 1.0 & 57 & 1.0 & $\mid 58$ & 1.0 & & & & \\
\hline 1.992 & \begin{tabular}{l|l}
62 & $\mathbf{9 9 7}$ \\
\end{tabular} & 63.99 & 64.98 & 65.99 & & 1080 & & 99 & & 1.0 & & & & \\
\hline
\end{tabular}

have manually obtained all 78 ROI's (ROI: region of interest), each of which includes one object of interest, from 51 test images presented in [18]. For each of the objects $\mathrm{C} 1$ to $\mathrm{C} 10$, we collect 500 images under similar but not identical imaging conditions. For each of these images, we also extract ranked SIFT features to construct a graph, using the same method presented in [5].

Using the trained model, these object images are recognized according to the following Steps:

1) Obtaining the KNNG for each graph;

2) Recognition by making using a majority voting strategy weighted by the similarities of the corresponding KNNG.

Suppose $N_{T P}$ is the number of correctly recognized instances for an object of interest, $N_{F P}$ is the number of instances incorrectly recognized as the object of interest, $N_{P}$ is the number of instances belonging to the object of interest. Then the F-measure is defined as follows:

$$
f=\frac{2}{1 / \text { recall }+1 / \text { precision }} \text {. }
$$

where recall $=\frac{N_{T P}}{N_{P}}$, precision $=\frac{N_{T P}}{N_{T P}+N_{F P}}$. The F-measures for the recognition test for Object 1 to Object 68 are shown in Table1. It is interesting to note that the test 
recognition performance of Object B1 to B8 is very close to that obtained in [18] when only SIFT features are used (corresponding to the 8 objects marked in magenta in Table 1. However, we emphasize that our results are obtained with large negative sample sets.

\section{Conclusion}

This paper has described a framework for learning recognition oriented exemplar models from a large corpus of multi-view images. Our model is a comprehensive integration of the global and local information contained in the local features from different views. The exemplars are extracted in a three-stage clustering process. First, RSOM tree clustering is used to incrementally cluster a large corpus of local invariant feature descriptors. Using RSOM, the K nearest neighbor graphs of each graph can be efficiently obtained without linear search. Second, the similarity propagation based graph clustering method is used to cluster the graph instances of a specific object with high precision. Such a graph cluster is termed an FTOG. Third, each FTOG is then subjected to affinity propagation to obtain the exemplars for a single FTOG. For each additional test graph, the recognition decision is made according to its nearest exemplar. Experiments demonstrate high performance in terms of efficiency, scalability and recognition.

\section{References}

1. Frey, B., Dueck, D.: Clustering by Passing Messages Between Data Points. Science 315(5814), 972-976 (2007)

2. Xia, S.P., Liu, J.J., Yuan, Z.T., Yu, H., Zhang, L.F., Yu., W.X.: Cluster-Computer Based Incremental and Distributed RSOM Data-Clustering. ACTA Electronica sinica. 35(3), 385-391 (2007)

3. Xia, S.P., Hancock, E.R.: 3D Object Recognition Using Hyper-Graphs and Ranked Local Invariant Features. In: da Vitoria Lobo, N., Kasparis, T., Roli, F., Kwok, J.T., Georgiopoulos, M., Anagnostopoulos, G.C., Loog, M. (eds.) S+SSPR 2008. LNCS, vol. 5342, pp. 117-126. Springer, Heidelberg (2008)

4. Xia, S.P., Hancock, E.R.: Pairwise Similarity Propagation Based Graph Clustering for Scalable Object Indexing and Retrieval. In: Torsello, A., Escolano, F., Brun, L. (eds.) GbRPR 2009. LNCS, vol. 5534, pp. 184-194. Springer, Heidelberg (2009)

5. Xia, S.P., Ren, P., Hancock, E.R.: Ranking the Local Invariant Features for the Robust Visual Saliencies. In: ICPR (2008)

6. Xia, S.P., Hancock, E.R.: Learning Class Specific Hypergraphs. In: Foggia, P., Sansone, C., Vento, M. (eds.) Image Analysis and Processing - ICIAP 2009. LNCS, vol. 5716, pp. 269-277. Springer, Heidelberg (2009)

7. Xia, S.P., Hancock, E.R.: Graph-Based Object Class Discovery. In: Jiang, X., Petkov, N. (eds.) Computer Analysis of Images and Patterns. LNCS, vol. 5702, pp. 385-393. Springer, Heidelberg (2009)

8. Bay, H., Tuytelaars, T., Gool, L.V.: SURF: Speeded Up Robust Features. In: Leonardis, A., Bischof, H., Pinz, A. (eds.) ECCV 2006. LNCS, vol. 3951, pp. 404-417. Springer, Heidelberg (2006)

9. Lowe, D.G.: Distinctive image features from scale-invariant key points. IJCV 60(2), 91-110 (2004) 
10. Chum, O., Philbin, J., Sivic, J., Isard, M., Zisserman, A.: Total recall: Automatic query expansion with a generative feature model for object retrieval. In: ICCV (2007)

11. Chung, F.: Spectral graph theory. American Mathematical Society, Providence (1997)

12. Everingham, M., Gool, L.V., Williams, C., Winn, J., Zisserman, A.: Overview and results of classification challenge. In: The PASCAL VOC 2007 Challenge Workshop, in conj. with ICCV (2007)

13. Li, F.F., Perona, P.: A Bayesian hierarchical model for learning natural scene categories. CVPR 2, 524-531 (2005)

14. Kadir, T., Brady, M., Zisserman, A.: An Invariant Method for Selecting Salient Regions in Images. In: Proc. Eighth ECCV., vol. 1(1), pp. 345-457 (2004)

15. Mikolajczyk, K., Schmid, C.: A Performance Evaluation of Local Descriptors. PAMI 27(10), 1615-1639 (2005)

16. Philbin, J., Chum, O., Isard, M., Sivic, J., Zissermans, A.: Object retrieval with large vocabularies and fast spatial matching. In: CVPR (2007)

17. Philbin, J., Sivic, J., Zisserman, A.: Geometric LDA: A Generative Model for Particular Object Discovery. BMVC (2008)

18. Rothganger, F., Lazebnik, S., Schmid, C., Ponce, J.: 3D Object Modeling and Recognition Using Local Affine-Invariant Image Descriptors and Multi-View Spatial Constraints. IJCV 66(3), 231-259 (2006)

19. Schonemann, P.H.: A generalized solution of the orthogonal Procrustes problem. Psychometrika 31(3), 1-10 (1966)

20. Sivic, J., Zisserman, A.: Video Google: A text retrieval approach to object matching in videos. In: ICCV, pp. 1470-1477 (2003) 